\title{
Growth Differentiation Factor-15 Levels at Admission Provide Incremental Prognostic Information on All- Cause Long-term Mortality in ST-Segment Elevation Myocardial Infarction Patients Treated with Primary Percutaneous Coronary Intervention
}

\author{
Mathijs C. Bodde - Maaike P. J. Hermans - Arnoud van der Laarse - Bart Mertens • \\ Fred P. H. T. M. Romijn · Martin J. Schalij · Christa M. Cobbaert · J. Wouter Jukema
}

Received: December 2, 2018 / Published online: January 30, 2019

(C) The Author(s) 2019

\section{ABSTRACT}

Introduction: To investigate the additive prognostic value of growth differentiation factor (GDF-15) levels in ST-segment elevation myocardial infarction (STEMI) patients treated with primary percutaneously coronary intervention (pPCI) with 10-year mortality on top of

Christa M. Cobbaert and J. Wouter Jukema share senior co-authorship.

Enhanced digital features To view enhanced digital features for this article go to https://doi.org/10.6084/ m9.figshare.7583345.

Electronic supplementary material The online version of this article (https://doi.org/10.1007/s40119019-0127-4) contains supplementary material, which is available to authorized users.

M. C. Bodde - M. P. J. Hermans - A. van der Laarse . M. J. Schalij · J. W. Jukema ( $₫)$

Department of Cardiology, Leiden University

Medical Center, Leiden, The Netherlands

e-mail: j.w.jukema@lumc.nl

A. van der Laarse · F. P. H. T. M. Romijn .

C. M. Cobbaert

Department of Clinical Chemistry and Laboratory

Medicine, Leiden University Medical Center,

Leiden, The Netherlands

\section{B. Mertens}

Department of Medical Statistics and

Bioinformatics, Leiden University Medical Center,

Leiden, The Netherlands clinical characteristics and known cardiac biomarkers.

Methods: Baseline serum GDF-15 levels were measured in 290 STEMI patients treated with pPCI in the MISSION! intervention trial conducted from February 1, 2004 through October 31,2006 . The incremental prognostic value of GDF-15 and NTproBNP levels was evaluated on top of clinical characteristics using Cox proportional hazards analysis, Chi-square models and C-index. Outcome was 10-year all-cause mortality.

Results: Mean age was $59.0 \pm 11.5$ years and 65 (22.4) patients were female. A total of 37 patients died during a follow-up of 9.4 (IQR 8.8-10.0) years. Multivariable Cox regression revealed GDF-15 and NTproBNP levels above median to be independently associated with 10-year all-cause mortality [HR GDF-15, 2.453 (95\% CI 1.064-5.658), $P=0.04$; HR NTproBNP, 2.413 (95\% CI 1.043-5.564), $P=0.04$ ] after correction for other clinical variables. Stratified by median GDF-15 (37.78 pmol/L) and NTproBNP (11.74 pmol/L) levels, Kaplan-Meier curves showed significant better survival for patients with GDF-15 and NTproBNP levels below the median versus above the median. The likelihood ratio test showed a significant incremental value of GDF-15 $(P=0.03)$ as compared with a model with clinically important variables and NTproBNP. The C-statistics for this model improved from 0.82 to 0.84 when adding GDF-15. 
Conclusion: GDF-15 levels at admission in STEMI patients are independently associated with 10-year all-cause mortality rates and could improve risk stratification on top of clinical variables and other cardiac biomarkers.

Keywords: GDF-15; Mortality; NTproBNP; Prognosis; Risk stratification; STEMI

\section{INTRODUCTION}

Long-term mortality rates in ST-segment elevation myocardial infarction (STEMI) patients treated with primary percutaneous coronary intervention (pPCI) are declining owing to more frequent use of reperfusion therapy, modern antithrombotic therapy and secondary prevention measures $[1,2]$. However, they are still substantial with a reported 1-year mortality rate of $10 \%[3,4]$ and a 5 -year mortality rate of about $23 \%$ [4].

Identification of high-risk patients is essential for optimal monitoring and initiation of appropriate treatment to reduce risk of events. Current risk assessment relies mainly on clinical characteristics such as age, infarct location, Killip class, cardiogenic shock, ejection fraction, diabetes, renal failure and time of ischemia [5-7]. In addition, traditional cardiac biomarkers such as cardiac troponin (cTn) and N-terminal pro-Btype natriuretic peptide (NTproBNP) have been shown to improve risk prediction in STEMI patients on top of clinical characteristics $[8,9]$.

Growth differentiation factor-15 (GDF-15) is a systemic stress-responsive member of the transforming growth factor beta (TGF- $\beta$ ) superfamily [10]. GDF-15 is a general, relatively novel biomarker which is induced in the myocardium after ischemia and reperfusion [11] and released as a result of haemodynamic stress [12]. However, GDF-15 is also released in the setting of inflammation or tissue damage and its overexpression has been found in a number of malignancies [11, 13].

Several studies have shown that GDF-15 levels in STEMI patients provide prognostic information on mortality rates within 1 year additional to established clinical and biochemical biomarkers [14-18]. Apart from this relatively short follow-up duration, these studies were limited since study populations were not always comparable because of differences with respect to timing of GDF-15 measurement, type of thrombolytic therapy and endpoints. So, whether GDF15 levels at admission are also related to longterm mortality rate is unknown in STEMI patients treated with pPCI, especially on top of other more recently validated biomarkers such as cTn and NTproBNP.

Therefore, the additive prognostic value of GDF-15 levels at admission in STEMI patients treated with pPCI as to 10-year mortality rate is investigated on top of clinical characteristics and known cardiac biomarkers such as cTn and NTproBNP.

\section{METHODS}

\section{Study Population}

In this study, data is used from patients with STEMI, who were included in the prospective MISSION! intervention trial [19]. The MISSION! intervention trial was conducted from February 2004 to October 2006. In this randomized study, we evaluated clinical and angiographic results in patients with STEMI treated with either bare metal stents (BMS) or sirolimus-eluting stents (SES) during pPCI. In short, patients were eligible if STEMI symptoms started less than $9 \mathrm{~h}$ before the procedure and the electrocardiogram (ECG) showed ST elevation (at least $0.2 \mathrm{mV}$ in at least two leads in V1-V3 or at least $0.1 \mathrm{mV}$ in other leads) or presumed new left bundle branch block (LBBB). Patients were excluded if they were aged less than 18 years or over 80 years. The study protocol was approved by the Medical Ethical Committee in the Leiden University Medical Center (LUMC). This study was conducted according to the declaration of Helsinki and written informed consent was obtained from all patients before enrolment in the study.

\section{Study Procedure}

During the study, all subsequent patients were treated according to the institutional MISSION! protocol [20], based on guidelines of the 
European Society of Cardiology, American College of Cardiology and the American Heart Association $[21,22]$. The pre-hospital protocol included diagnosis by field triage by 12-lead ECG and in-ambulance treatment with a loading dose of clopidogrel, aspirin, heparin and intravenous glycoprotein IIb/IIIa inhibitors. pPCI was performed according to the clinical guidelines [21, 22]. If tolerated, patients received beta-blockers, ACE inhibitors and statins within $24 \mathrm{~h}$. Additionally, patients were prescribed dual antiplatelet therapy, consisting of aspirin $100 \mathrm{mg}$ daily for life and clopidogrel $75 \mathrm{mg}$ daily for 12 months. More than $95 \%$ of the patients received a statin, an acetylsalicylic acid, and a thienopyridine and more than 85\% of the patients received a beta-blocker and an ACE inhibitor within $24 \mathrm{~h}$ after admission. During admission, patients' demographic characteristics, risk factors and clinical features were collected. Clinical follow-up data was collected during the outpatient clinic visits at 30 days, 3 , 6 and 12 months. Information on all-cause mortality was obtained from the Dutch Municipality Records registry at 2, 5 and 10 years after admission. Cause of death was retrieved from general practitioners. The primary outcome of this analysis was 10-year all-cause mortality.

\section{GDF-15 and NTproBNP Measurement}

GDF-15 and NTproBNP levels are expressed in picomoles per litre which represents the amount of substances. GDF-15 levels are converted to nanograms per litre by dividing the number by 0.02929 . NTproBNP levels are converted to nanograms per litre by dividing the number by 0.118 .

Blood samples were obtained at presentation before the pPCI procedure was performed. An extra serum sample was coagulated for at least 60 min before centrifugation at 1500 relative centrifugal force (RCF) for $10 \mathrm{~min}$ at $18{ }^{\circ} \mathrm{C}$. Sera were pipetted into $1.1-\mathrm{mL}$ Micronic tubes. Within $2 \mathrm{~h}$ after vena puncture, the serum samples were frozen at $-70 /-80^{\circ} \mathrm{C}$ in a freezer.

For the in vitro quantitative determination of GDF-15 in human serum, a Roche electrochemiluminescence immunoassay (ECLIA) on Cobas e602 series (catalogue number 07125933190) is used (Roche Diagnostics, Mannheim, Germany). The test is based on the sandwich principle. Results are determined via a calibration curve which is instrument-specifically generated by two-point calibration and a master curve provided via the reagent barcode.

Serum fractions of $250 \mu \mathrm{L}$ were used for parallel quantification of both serum GDF-15 and NTproBNP. GDF-15 values below the limit of detection were reported as less than $11.71 \mathrm{pmol} / \mathrm{L}$. Values exceeding the measuring range were reported as greater than $585.8 \mathrm{pmol} / \mathrm{L}$.

All GDF-15 measurements were performed at Leiden University Medical Center by investigators who were not aware of patients' characteristics and outcomes.

\section{NTproBNP Measurements}

A Roche ECLIA on Cobas e602 series (catalogue number 07125933190) is used (Roche Diagnostics, Mannheim, Germany) to determine NTproBNP levels. The test is based on the sandwich principle and has a detection limit of $0.59 \mathrm{pmol} / \mathrm{L}$. Values exceeding the measuring range were reported as greater than $4130 \mathrm{pmol} /$ L. Results are determined via a calibration curve which is instrument-specifically generated by two-point calibration and a master curve provided via the reagent barcode.

\section{Statistical Analysis}

Normally distributed data is presented as mean and standard deviation (SD). Non-normally distributed data is expressed as median with interquartile range (IQR). Categorical data is expressed as absolute numbers and percentages. Differences in baseline characteristics between patients below and above the median of GDF-15 were assessed with an independent $T$ test, the Mann-Whitney $U$ test or Chi-square test when appropriate. The Pearson correlation coefficient was used to analyse the correlation between GDF-15 and cTnT and NT-proBNP. Event-free survival was analysed with Kaplan-Meier curves 
and compared between groups with the logrank test. To assess the incremental value of GDF-15 levels at admission, we first investigated independent univariate Cox regression analyses to determine the association of potential confounding variables, like body mass index (BMI), hypertension, diabetes, hypercholesterolaemia, smoking, history of cardiovascular disease (CVD), prior MI, out of hospital cardiac arrest, cardiogenic shock, culprit vessel, number of vessel disease, type of stent, cTnT, creatine kinase (CK) and creatinine, on 10-year all-cause mortality. We then constructed a base multivariate Cox model which adjusts for age, gender and all variables with a $P<0.10$ from the univariable analysis. The incremental prognostic value of GDF-15 and NTproBNP levels was then evaluated by independently adding these predictors to the base model and calculating the likelihood ratio for addition of these effects on top of clinical variables. In addition, the overall C-statistic as proposed by Harrell et al. [23] was calculated. Effects are reported as hazard ratios (HR) with 95\% confidence intervals (CI). All statistical tests were performed with SPSS software (Version 24.0, IBM, Armonk, NY, USA). $P$ values less than 0.05 assessed by two-sided tests were considered to be statistically significant.

\section{RESULTS}

\section{Baseline and Clinical Characteristics}

Baseline serum GDF-15 levels were available in 290 STEMI patients treated with pPCI in the MISSION! intervention trail. Mean age was $59.0 \pm 11.5$ years, 65 (22.4) patients were female and the median GDF-15 concentration was $37.78 \mathrm{pmol} / \mathrm{L}$ (IQR 26.88-55.83 pmol/L). Stratified by median GDF-15 levels, patients with values above the median were older, more often female and had higher NTproBNP, cTnT, creatine kinase $(\mathrm{CK})$ and creatinine levels than the patients below the median (Table 1). The correlation coefficient between GDF-15 and NTproBNP was $0.17(P=0.004)$ and that between GDF-15 and cTnT was $0.083(P=0.191)$.

\section{Long-Term Clinical Outcome}

A total of 37 patients reached the endpoint during a follow-up of 9.4 (IQR 8.8-10.0) years. The cause of death was adjudicated as cardiac origin in 10 patients, 4 patients died of likely cardiac origin, 19 patients died from a noncardiac cause and the cause of death is unknown in 4 patients.

\section{Survival Analysis}

Univariable Cox regression analysis showed that age, diabetes, current smokers, patients with a family history of CVD, cardiogenic shock, more than one vessel disease at the time of STEMI, baseline levels of GDF-15 above the median, baseline NTproBNP level above the median $(11.74 \mathrm{pmol} / \mathrm{L})$, and renal dysfunction were associated with unfavourable outcome (Table 2). Infarct size expressed by the biomarkers cTnT and area under the curve of CK were not associated with higher mortality rates. Multivariable Cox regression revealed that GDF15 and NTproBNP levels above median are independently associated with 10-year all-cause mortality [HR GDF-15, $2.453 \quad(95 \%$ CI 1.064-5.658), $P=0.04$; HR NTproBNP, 2.413 (95\% CI 1.043-5.586), $P=0.04$ ] after correction for clinical variables. Furthermore, age [HR 1.095 (95\% CI 1.044-1.150), $P<0.001]$ and cardiogenic shock [HR $13.062 \quad(95 \%$ CI 3.374-50.566), $P<0.001]$ remained significantly associated with all-cause mortality in the multivariable Cox regression analysis.

Stratified by median GDF-15 (37.78 pmol/L) and median NTproBNP (11.74 pmol/L) levels, Kaplan-Meier curves showed significantly better survival for patients with GDF-15 and NTproBNP levels below the median than for patients with GDF-15 and NTproBNP levels above the median. In the group with GDF-15 levels below the median, the event-free survival was $92.6 \%$, compared to $79.8 \%$ in the group with GDF-15 levels above the median (log-rank $P<0.001$ ) (Fig. 1a). Similar results were obtained with NTproBNP levels. The event-free survival rate was $93.5 \%$ with NTproBNP levels below the median, versus $78.8 \%$ event-free 
Table 1 Demographic and clinical characteristics at baseline

\begin{tabular}{|c|c|c|c|c|}
\hline \multirow[t]{2}{*}{ Variable } & \multirow[t]{2}{*}{$\begin{array}{l}\text { Total group } \\
(n=290)\end{array}$} & \multicolumn{2}{|c|}{$\begin{array}{l}\text { Baseline GDF-15 median }(37.8 \mathrm{pmol} / \\
\text { L) }\end{array}$} & \multirow[t]{2}{*}{$P$} \\
\hline & & $\begin{array}{l}\text { median } \\
(n=145)\end{array}$ & $\begin{array}{l}>\text { median } \\
(n=145)\end{array}$ & \\
\hline Age, mean (SD), years & $59.0(11.5)$ & $55.7(11.8)$ & $62.4(10.9)$ & $<0.001$ \\
\hline Female gender, $n(\%)$ & $65(22.4)$ & $18(12.4)$ & $47(32.4)$ & $<0.001$ \\
\hline \multicolumn{5}{|l|}{ Cardiovascular risk factors } \\
\hline Current smoking & $158(54.5)$ & $82(56.6)$ & $76(52.4)$ & 0.48 \\
\hline Ex-smoker & $33(11.4)$ & $17(11.7)$ & $16(11.0)$ & 0.85 \\
\hline NIDDM, $n(\%)$ & $19(6.6)$ & $6(4.1)$ & $13(9.0)$ & 0.10 \\
\hline IDDM, $n(\%)$ & $11(3.7)$ & $6(4.1)$ & $5(3.4)$ & 0.76 \\
\hline Family history of CVD, $n(\%)$ & $126(43.4)$ & $70(48.3)$ & $56(38.6)$ & 0.17 \\
\hline Treated hypercholesterolaemia, $n$ (\%) & $56(19.3)$ & $25(17.2)$ & $31(21.4)$ & 0.37 \\
\hline Treated hypertension, $n(\%)$ & $82(28.3)$ & $41(28.3)$ & $41(28.3)$ & 1.00 \\
\hline Body mass index, mean (SD), $\mathrm{kg} / \mathrm{m}^{2}$ & $26.6(4.2)$ & $26.7(3.8)$ & $26.6(4.6)$ & 0.87 \\
\hline \multicolumn{5}{|l|}{ Comorbidities } \\
\hline Previous myocardial infarction, $n$ (\%) & $11(3.8)$ & $6(4.1)$ & $5(3.4)$ & 0.76 \\
\hline Previous PCI, $n(\%)$ & $5(1.7)$ & $3(2.1)$ & $2(1.4)$ & 0.65 \\
\hline Previous CABG, $n(\%)$ & $2(0.7)$ & $2(1.4)$ & $0(-)$ & 0.16 \\
\hline History of cerebrovascular disease, $n(\%)$ & $10(3.4)$ & $3(2.1)$ & $7(4.8)$ & 0.27 \\
\hline \multicolumn{5}{|l|}{ Previous medication use } \\
\hline Beta-blocker, $n(\%)$ & $36(12.4)$ & $20(13.8)$ & $16(11.0)$ & 0.48 \\
\hline ACE inhibitor/AT2 antagonist, $n(\%)$ & $34(11.7)$ & $19(13.1)$ & $15(10.3)$ & 0.47 \\
\hline Statin, $n(\%)$ & $31(10.7)$ & $14(9.7)$ & $17(11.7)$ & 0.51 \\
\hline Antiplatelet, $n(\%)$ & $1(0.3)$ & $1(0.7)$ & $0(-)$ & 0.37 \\
\hline Ascal, $n(\%)$ & $28(9.7)$ & $11(7.6)$ & $17(11.7)$ & 0.29 \\
\hline \multicolumn{5}{|l|}{ Clinical characteristics } \\
\hline Time of ischemia, median (IQR), min & $192(146-257)$ & $200(147-260)$ & $191(146-248)$ & 0.58 \\
\hline Number of narrowed coronary arteries & & & & 0.99 \\
\hline 1 & $158(54.5)$ & $78(53.8)$ & $80(55.2)$ & \\
\hline 2 & $115(39.7)$ & $58(40.0)$ & $57(39.3)$ & \\
\hline 3 & $15(5.2)$ & $8(5.5)$ & $7(4.8)$ & \\
\hline Complete revascularization, $n(\%)$ & $195(67.5)$ & $101(71.1)$ & $94(65.7)$ & 0.38 \\
\hline Killip class $\geq 2, n(\%)$ & $27(9.3)$ & $11(7.6)$ & $16(11.0)$ & 0.37 \\
\hline
\end{tabular}


Table 1 continued

\begin{tabular}{|c|c|c|c|c|}
\hline \multirow[t]{2}{*}{ Variable } & \multirow[t]{2}{*}{$\begin{array}{l}\text { Total group } \\
(n=290)\end{array}$} & \multicolumn{2}{|c|}{$\begin{array}{l}\text { Baseline GDF-15 median ( } 37.8 \mathrm{pmol} / \\
\text { L) }\end{array}$} & \multirow[t]{2}{*}{$P$} \\
\hline & & $\begin{array}{l}<\text { median } \\
(n=145)\end{array}$ & $\begin{array}{l}>\text { median } \\
(n=145)\end{array}$ & \\
\hline \multicolumn{5}{|l|}{ Laboratory results } \\
\hline $\begin{array}{l}\text { Infarct size, median area under the CK curve } \\
(\mathrm{IQR}), \mathrm{g} / \mathrm{m}^{2}\end{array}$ & $8.92(4.26-15.82)$ & $7.36(2.93-14.45)$ & $10.54(5.73-16.99)$ & 0.009 \\
\hline Peak cardiac troponin-T, median (IQR), $\mu \mathrm{g} / \mathrm{L}$ & $5.53(2.28-10.22)$ & $4.77(1.64-8.84)$ & $5.91(3.08-10.72)$ & 0.02 \\
\hline NTproBNP, median (IQR), pmol/L & $11.74(4.70-27.53)$ & $9.46(4.33-22.98)$ & $14.49(5.40-34.24)$ & 0.02 \\
\hline Creatinine, mean (SD), $\mu \mathrm{mol} / \mathrm{L}$ & $81.6(18.5)$ & $78.2(13.8)$ & $85.1(21.8)$ & 0.002 \\
\hline
\end{tabular}

Data are expressed as number (\%), median (IQR) or mean \pm standard deviation

Narrowed coronary artery, defined as $\geq 50 \%$ stenosis on baseline coronary angiogram. Treated hypercholesterolaemia, serum total cholesterol $\geq 6 \mathrm{mmol} / \mathrm{L}$ and/or serum $\mathrm{TG} \geq 2.2 \mathrm{mmol} / \mathrm{L}$ or treatment with lipid -lowering drugs. Treated hypertension, defined as systolic blood pressure $\geq 140 \mathrm{mmHg}$ and/or diastolic blood pressure $\geq 90 \mathrm{mmHg}$ and/or the use of antihypertensive medication

$A C E$ angiotensin-converting enzyme, $A T 2$ angiotensin II, $C A B G$ coronary artery bypass surgery, $C V D$ cardiovascular disease, $G D F-15$ growth differentiation factor-15, IDDM insulin-dependent diabetes mellitus, $I Q R$ interquartile range, NIDDM non-insulin-dependent diabetes mellitus, $N T$ proBNP $\mathrm{N}$-terminal pro b-type natriuretic peptide, $P C I$ percutaneous coronary intervention, $S D$ standard deviation

survival in patients with an NTproBNP level above the median (log rank $P<0.001)$. When these biomarkers are divided in four groups (both GDF-15 and NTproBNP < median; GDF$15>$ median and NTproBNP $<$ median; GDF$15<$ median and NTproBNP $>$ median; and both GDF-15 and NTproBNP > median), the prognostic value improved. In the group with both GDF-15 and NTproBNP levels below their medians the event-free survival was $95.7 \%$, whereas in the group with both GDF-15 and NTproBNP levels above their medians (log rank $P<0.001)$ the event-free survival was $70.7 \%$ (Fig. 2).

\section{Incremental Value of GDF-15}

Figure 3 shows the incremental value of NTproBNP and GDF-15 on top of other clinically important risk factors for predicting the primary endpoint. Model 1 includes all variables that are significant in the univariable Cox regression analysis. The addition of NTproBNP to the basic model improved the likelihood ratio but this was not statistically significant $(P=0.086)$. The likelihood ratio test showed a significantly incremental value of GDF-15 $(P=0.027)$ as compared with a model with clinical important variables and NTproBNP. The C-statistics for this model improved from 0.82 to 0.84 when adding GDF-15 levels to the model with all clinically important risk factors and NTproBNP levels.

\section{DISCUSSION}

This study demonstrates that higher GDF-15 levels at admission are associated with 10-year all-cause mortality in STEMI patients treated with pPCI. This relation was independent of clinical risk factors and biomarkers. Moreover, GDF-15 levels at admission have additional prognostic value beyond identified risk factors and other cardiac biomarkers such as cTn and NTproBNP as analysed by Chi-square test and C-statistics.

When stratified by median GDF-15 levels, patients with a level below the median show an 
Table 2 Univariable and multivariable Cox proportional hazard regression analysis to identify independent predictors of all-cause mortality

\begin{tabular}{|c|c|c|c|c|}
\hline Parameter & $\begin{array}{l}\text { Univariable analysis } \\
\text { HR ( } 95 \% \text { CI) }\end{array}$ & $P$ & $\begin{array}{l}\text { Multivariable analysis } \\
\text { HR ( } 95 \% \text { CI })\end{array}$ & $P$ \\
\hline Age, mean, years & $1.110(1.069-1.153)$ & $<0.001$ & $1.095(1.044-1.150)$ & $<0.001$ \\
\hline Female gender & $0.761(0.334-1.733)$ & 0.51 & $0.413(0.159-1.072)$ & 0.07 \\
\hline Body mass index, mean, $\mathrm{kg} / \mathrm{m}^{2}$ & $0.962(0.881-1.050)$ & 0.39 & & \\
\hline Treated hypertension & $0.957(0.463-1.978)$ & 0.91 & & \\
\hline Diabetes & $2.176(0.956-4.954)$ & 0.06 & $2.374(0.997-5.654)$ & 0.05 \\
\hline Treated hypercholesterolaemia & $0.875(0.365-2.098)$ & 0.765 & & \\
\hline Current smoker & $0.576(0.299-1.109)$ & 0.10 & $1.702(0.821-3.525)$ & 0.15 \\
\hline Family history of CVD & $0.453(0.219-0.936)$ & 0.03 & $0.871(0.384-1.974)$ & 0.74 \\
\hline Prior myocardial infarction & $1.322(0.318-5.500)$ & 0.70 & & \\
\hline Out of hospital cardiac arrest & $0.048(0.000-568.83)$ & 0.53 & & \\
\hline Cardiogenic shock & $10.76(3.295-35.15)$ & $<0.001$ & $13.062(3.374-50.566)$ & $<0.001$ \\
\hline \multicolumn{5}{|l|}{ Culprit vessel } \\
\hline RCA & Ref & 0.65 & & \\
\hline $\mathrm{RCX}$ & $0.944(0.359-2.484)$ & 0.91 & & \\
\hline LAD & $0.727(0.356-1.483)$ & 0.38 & & \\
\hline Number of vessel disease $(>50 \%)>1$ & $1.914(0.993-3.689)$ & 0.05 & $1.383(0.457-4.183)$ & 0.57 \\
\hline Complete revascularization & $0.480(0.252-0.915)$ & 0.026 & $0.977(0.329-2.095)$ & 0.97 \\
\hline Drug-eluting stent & $1.256(0.657-2.412)$ & 0.49 & & \\
\hline Peak cardiac troponin-T level, $\mu \mathrm{g} / \mathrm{L}$ & $1.023(0.973-1.076)$ & 0.37 & & \\
\hline $\begin{array}{l}\text { Infarct size, median under the CK } \\
\text { curve (IQR), } \mathrm{g} / \mathrm{m}^{2}\end{array}$ & $0.979(0.940-1.019)$ & 0.31 & & \\
\hline Baseline GDF-15 > median & $3.360(1.585-7.121)$ & 0.002 & $2.453(1.064-5.658)$ & 0.04 \\
\hline Baseline NTproBNP $>$ median & $3.332(1.567-7.042)$ & 0.002 & $2.413(1.043-5.586)$ & 0.04 \\
\hline Creatinine, $\mu \mathrm{mol} / \mathrm{L}$ & $1.023(1.007-1.039)$ & 0.004 & $1.005(0.988-1.022)$ & 0.56 \\
\hline
\end{tabular}

Data are expressed as hazard ratios with $95 \%$ confidence interval

Treated hypercholesterolaemia, serum total cholesterol $\geq 6 \mathrm{mmol} / \mathrm{L}$ and/or serum TG $\geq 2.2 \mathrm{mmol} / \mathrm{L}$ or treatment with lipid-lowering drugs. Treated hypertension, defined as systolic blood pressure $\geq 140 \mathrm{mmHg}$ and/or diastolic blood pressure $\geq 90 \mathrm{mmHg}$ and/or the use of antihypertensive medication

$C K$ creatine kinase, $C V D$ cardiovascular disease, GDF-15 growth differentiation factor-15, NTproBNP N-terminal pro b-type natriuretic peptide, $S D$ standard deviation

excellent prognosis with a 10-year all-cause mortality rate of $6.2 \%$ compared to $19.3 \%$ in the group with a GDF-15 level above the median. NTproBNP levels stratified by the median show the same division of 10-year allcause mortality rates as GDF-15 levels. However, 


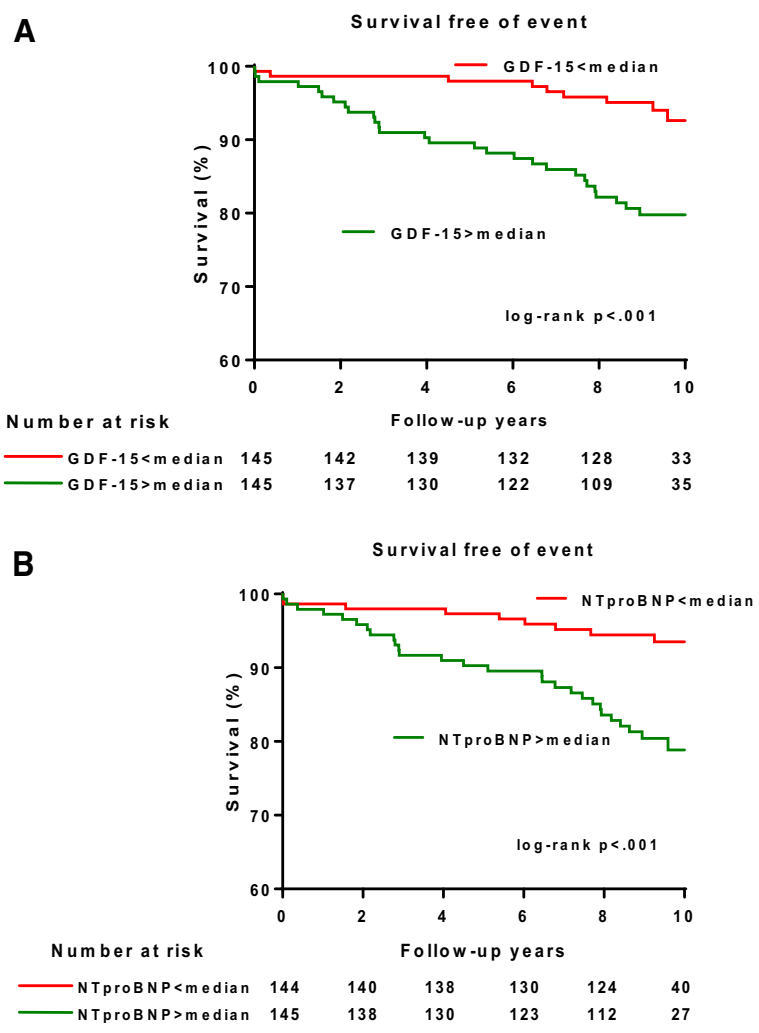

Fig. 1 Kaplan-Meier analysis to evaluate the survival free of the primary endpoint of all-cause mortality. a GDF-15. b NTproBNP. GDF-15 growth differentiation factor-15, NTproBNP N-terminal pro-B-type natriuretic peptide

combining these two biomarkers reveals that they have a complementary relation with 10 -year all-cause mortality. So, the combination of these biomarkers seems to identify an interesting group of high-risk patients. In the group of patients with both GDF-15 and NTproBNP levels below the median, only 3 patients $(3.8 \%)$ died within 10 years compared to $22(27.8 \%)$ in the group with both GDF-15 and NTproBNP levels above the median. Furthermore, GDF-15 shows additional prognostic information when added to clinical information and NTproBNP.

Other studies in a broad spectrum of patients have shown that a high level of GDF-15 is independently associated with mortality and adds extra prognostic value on top of various clinical characteristics and biomarkers [12, 14-18, 24-26]. Several of these studies compared the GDF-15 levels of STEMI patients at admission with outcome [14-16]. Two of these studies used a comparable population with GDF-15 levels at admission of STEMI patients treated with pPCI. Eitel et al. demonstrated that GDF-15 levels at admission are a strong predictor of mortality after 6 years [16]. However, two important biomarkers, cTn and NTproBNP, were not available is this cohort. Recently, Velders et al. showed in a large cohort that GDF-15 is independently associated with cardiovascular death after 1 year after adjusting for these biomarkers, the severity of cardiovascular disease and other clinical information [14]. However, the number of studies that compared GDF-15 and NTproBNP levels at admission with all-cause mortality on the long term in STEMI patients treated with pPCI is virtually absent. To our knowledge the current study is the first that investigated these biomarkers at admission in relation to 10-year all-cause mortality in STEMI patients treated with pPCI.

A relatively low number of patients died in this cohort during the 10 years. In the first year of follow-up after STEMI only 5 patients (1.7\%) died, of whom 4 died from a cardiac cause. In the years that followed, 32 more patients died, of whom 6 died from a cardiac cause and 4 patients' deaths were likely of cardiac origin. In total 19 patients died of a non-cardiac cause, of which 18 were after more than 1 year. By adding biomarkers that reflect a more general state of disease, one might not only capture patients prone to cardiovascular events but also patients prone to non-cardiac events.

Current risk assessment with for example the TIMI or GRACE risk scores is mainly based upon clinical characteristics [14]. Cardiac biomarkers such as cTn and NTproBNP have been shown to improve risk prediction [8, 9]. Using a multimarker strategy that captures a broader spectrum of diseases may have added value since it can reveal novel release mechanisms and therefore potential therapeutic targets. The relation of GDF-15 to cTn and NTproBNP has shown that GDF-15 is involved in cardiac pathologies. GDF-15 is induced in the myocardium after ischemia [11]. Several studies have demonstrated that plasma levels of GDF15 , just as NTproBNP, are associated with mechanical stretch, left ventricular mass, 


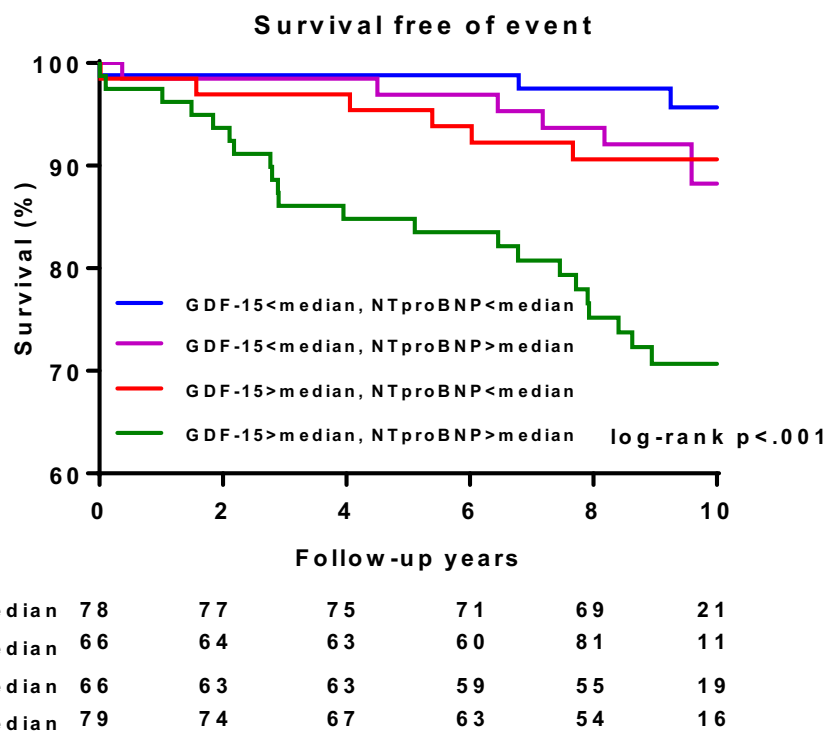

Fig. 2 Kaplan-Meier analysis to evaluate the survival free of experiencing the primary endpoint of all-cause mortality when combining assessment of GDF-15 and NTproBNP.

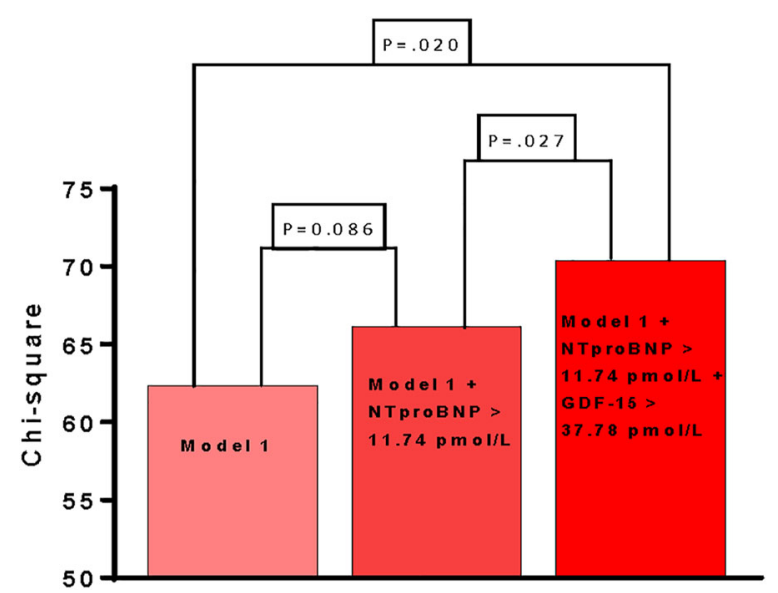

Fig. 3 The bar graphs show the incremental value of NTproBNP and GDF-15 on top of other clinically important risk factors for predicting the primary endpoint. Harrell C-statistics represent overall adequacy of the risk prediction. Model 1: Clinical variables (age, gender, previous diabetes mellitus, current smoking, family history of CVD, cardiogenic shock, $>1$ number of vessel disease, complete revascularization, creatinine). Model 2: Model $1+\mathrm{NT}$ proBNP $>$ median $(11.74 \mathrm{pmol} / \mathrm{L})$. Model 3: Model $1+$ NTproBNP $>$ median $(11.74 \mathrm{pmol} / \mathrm{L})+$ GDF-15 > median (37.78 pmol/L). CVD cardiovascular disease, GDF-15 growth differentiation factor-15, NTproBNP N-terminal pro-B-type natriuretic peptide
GDF-15 growth differentiation factor-15, NTproBNP $\mathrm{N}$-terminal pro-B-type natriuretic peptide

concentric left ventricular hypertrophy, reduced left ventricular ejection fraction, and heart failure $[12,15,27]$. Besides these similarities, the characteristics of GDF-15 release are distinct from those of NTproBNP and cTn. Earlier studies demonstrated that cTn release shows a typical rise and fall pattern, indicating the release from dying cardiomyocytes, whereas GDF-15 values increase within hours after ischemia [11] but remain remarkably stable over time during admission [28] and during 6 months of follow-up [24]. GDF-15 follows several stress pathways that differ from the cardiac-specific biomarkers, like NTproBNP and cTn [11, 29]. Plasma levels of GDF-15 may increase in response to pathological stress associated with vascular inflammation, endothelial activation or tissue damage, and its overexpression has been found in several malignancies [11, 24, 27, 30, 31]. Supported by epidemiological studies, this indicates that GDF-15 seems to be a marker of chronic cardiac and vascular pathologies, and is not per se related to acute injury $[27,32]$.

Over the last decade, a substantial amount of research has been performed that studied the role of GDF-15 levels for the risk assessment in acute coronary syndrome patients. However, 
translation of novel biomarkers into clinical practice has been shown to be challenging. For example, after the discovery of NTproBNP as a marker for heart failure it took two decades to implement it into clinical practice [33]. Recently a multidisciplinary working group defined a strategy and checklist to better identify the clinical unmet needs and how novel biomarkers can satisfy them [34]. They provide a practical approach to help assess whether a new biomarker would provide clinical benefit. To give a purely hypothetical example, we designed a map for a clinical pathway using this checklist and clinical approach (supplemental figure) on how we could advance GDF-15 into clinical practice. In this figure we used GDF-15 as an add-on test on top of clinical characteristics and cTn and NTproBNP. We acknowledge that we cannot implement GDF-15 into clinical practice solely on the basis of the results of our study, especially since it is currently unclear how GDF-15 levels can be lowered by medical therapy or interventions and whether lowering these levels results in an improved outcome. However we do hope that we can encourage other stakeholders to follow this example in larger prospective intervention studies to target this unmet clinical need with the development and implementation of GDF-15 in clinical pathway mapping.

Before this can be considered, further research with regard to the pathophysiological mechanisms and the influence of common and novel medical therapies on plasma levels of GDF-15 should be explored. Two potentially ways to do so might be more aggressive lipidlowering therapies or by anti-inflammatory therapy. The only study so far that investigated the relation between lipid lowering therapy by statin therapy and GDF-15 levels was conducted by Bonaca et al. [35]. They found no interaction of GDF-15 with different kind of statins and, moreover, GDF-15 levels did not decline after 4 months. Whether more aggressive medical therapy by for example PCSK-9 inhibitors has beneficial effects on GDF-15 is worth investigating. Another way to influence GDF-15 levels may be by anti-inflammatory therapy. In the recently published CANTOS (Canakinumab Anti-inflammatory Thrombosis Outcome
Study) trial it was shown that targeting the innate immunity pathway with canakinumab, independent of lipid level lowering, led to a significant lower rate of recurrent cardiovascular events than placebo [36]. It would be of interest to explore whether GDF-15 levels may act as biomarker-guided therapy to evaluate the effect of anti-inflammatory therapy.

Several limitations need to be mentioned. First of all, this study was a single-centre randomized trial with a limited number of patients; so, the results are limited to the patients eligible for the trial, although the results apply for a broad range of STEMI patients. All patients were treated according to the institutional MISSION! which provided an integrated approach of MI care to optimize treatment. This yielded a very homogeneous STEMI population. Secondly, limited data is available about the stability of GDF-15 samples after long-term storage at $-80^{\circ} \mathrm{C}$. However, an earlier study conducted by Daniels et al. [37] measured GDF-15 in 1391 serum samples that were stored for 14-18 years. The fact that GDF-15 levels were prognostic for outcome supports that there is sufficient stability to preserve a clinical signal [37]. Moreover, the median of the GDF-15 levels in the present cohort was $37.78 \mathrm{pmol} / \mathrm{L}$ which is comparable with the median levels of GDF-15 of $38.63 \mathrm{pmol} / \mathrm{L}$ in a similar cohort of STEMI patients [16]. Third, a relatively low number of events were noted during follow-up. To make the results more robust, larger cohorts of STEMI patients treated with pPCI should be followed. An explanation for the low number of events could be the exclusion criteria of patients older than 80 years. This could have led to a relatively young cohort with a mean age of 59 years. The last issue that should be addressed is the low number of events in relation to the multivariate Cox model. This paper has investigated the added value of GDF-15 for the prediction of 10-year all-cause mortality in addition to established risk factors as well as potential confounding variables identified from univariate analysis on our data. As opposed to fitting a prognostic model, a testing procedure was used, which first estimates a multivariate Cox model to account for the joint effect of these risk factors and potential confounders, after which the 
added value was assessed from likelihood ratio testing for the addition of GDF-15 and NTproBNP to the base model.

\section{CONCLUSIONS}

Baseline GDF-15 levels are independently associated with 10-year all-cause mortality rates and improve long-term risk stratification in STEMI patients treated with pPCI on top of clinical variables and other cardiac biomarkers. Before implementation into clinical practice can be considered, the clinical utility needs to be further validated in prospective intervention studies.

\section{ACKNOWLEDGEMENTS}

We thank W.H. Bax for her support collecting blood samples to measure GDF-15 and NTproBNP levels for this project. We thank the participants of the study.

Funding. The Department of Clinical Chemistry \& Laboratory Medicine received financial support from Roche Diagnostics to measure GDF-15 and NTproBNP levels. No funding was provided for article processing charges.

Authorship. All named authors meet the International Committee of Medical Journal Editors (ICMJE) criteria for authorship for this article, take responsibility for the integrity of the work as a whole, and have given their approval for this version to be published.

Disclosures. Mathijs Bodde, Maaike Hermans, Arnaud van der Laarse, Bart Mertens, Fred Romijn, Martin Schalij and Christa Cobbaert declare that they have nothing to disclose. Wouter Jukema is a member of the journal's editorial board.

Compliance with Ethics Guidelines. The study protocol was approved by the Medical Ethical Committee in the Leiden University Medical Center(LUMC). This study was conducted according to the declaration of Helsinki and written informed consent was obtained from all patients before enrolment in the study.

Data Availability. The datasets generated during and/or analyzed during the current study are not publicly available due to bad experiences regarding data hustling and cherry picking, but requests for collaborations are certainly welcome.

Open Access. This article is distributed under the terms of the Creative Commons Attribution-NonCommercial 4.0 International License (http://creativecommons.org/licenses/ by-nc/4.0/), which permits any noncommercial use, distribution, and reproduction in any medium, provided you give appropriate credit to the original author(s) and the source, provide a link to the Creative Commons license, and indicate if changes were made.

\section{REFERENCES}

1. Gale CP, Allan V, Cattle BA, et al. Trends in hospital treatments, including revascularisation, following acute myocardial infarction, 2003-2010: a multilevel and relative survival analysis for the National Institute for Cardiovascular Outcomes Research (NICOR). Heart. 2014;100(7):582-9.

2. Puymirat E, Simon T, Steg PG, et al. Association of changes in clinical characteristics and management with improvement in survival among patients with ST-elevation myocardial infarction. JAMA. 2012;308(10):998-1006.

3. Fokkema ML, James SK, Albertsson P, et al. Population trends in percutaneous coronary intervention: 20-year results from the SCAAR (Swedish Coronary Angiography and Angioplasty Registry). J Am Coll Cardiol. 2013;61(12):1222-30.

4. Pedersen F, Butrymovich V, Kelbaek H, et al. Shortand long-term cause of death in patients treated with primary PCI for STEMI. J Am Coll Cardiol. 2014;64(20):2101-8.

5. Chang WC, Kaul P, Fu Y, et al. Forecasting mortality: dynamic assessment of risk in ST-segment elevation acute myocardial infarction. Eur Heart J. 2006;27(4):419-26. 
6. Morrow DA, Antman EM, Charlesworth A, et al. TIMI risk score for ST-elevation myocardial infarction: a convenient, bedside, clinical score for risk assessment at presentation: an intravenous nPA for treatment of infarcting myocardium early II trial substudy. Circulation. 2000;102(17):2031-7.

7. Ibanez B, James S, Agewall S, et al. 2017 ESC guidelines for the management of acute myocardial infarction in patients presenting with ST-segment elevation: the Task Force for the management of acute myocardial infarction in patients presenting with ST-segment elevation of the European Society of Cardiology (ESC). Eur Heart J. 2018;39(2):119-77.

8. Bjorklund E, Jernberg T, Johanson P, et al. Admission N-terminal pro-brain natriuretic peptide and its interaction with admission troponin $\mathrm{T}$ and ST segment resolution for early risk stratification in ST elevation myocardial infarction. Heart. 2006;92(6):735-40.

9. O'Donoghue ML, Morrow DA, Cannon CP, et al. Multimarker risk stratification in patients with acute myocardial infarction. J Am Heart Assoc. 2016;5(5):e002586.

10. Bootcov MR, Bauskin AR, Valenzuela SM, et al. MIC-1, a novel macrophage inhibitory cytokine, is a divergent member of the TGF-beta superfamily. Proc Natl Acad Sci USA. 1997;94(21):11514-9.

11. Kempf T, Eden M, Strelau J, et al. The transforming growth factor-beta superfamily member growthdifferentiation factor-15 protects the heart from ischemia/reperfusion injury. Circ Res. 2006;98(3):351-60.

12. Chan MM, Santhanakrishnan R, Chong JP, et al. Growth differentiation factor 15 in heart failure with preserved vs. reduced ejection fraction. Eur J Heart Fail. 2016;18(1):81-8.

13. Wollert KC, Kempf T. Growth differentiation factor 15 in heart failure: an update. Curr Heart Fail Rep. 2012;9(4):337-45.

14. Velders MA, Wallentin L, Becker RC, et al. Biomarkers for risk stratification of patients with ST-elevation myocardial infarction treated with primary percutaneous coronary intervention: insights from the platelet inhibition and patient outcomes trial. Am Heart J. 2015;169(6):879-889.e7.

15. Khan SQ, Ng K, Dhillon O, et al. Growth differentiation factor-15 as a prognostic marker in patients with acute myocardial infarction. Eur Heart J. 2009;30(9):1057-65.

16. Eitel I, Blase P, Adams V, et al. Growth-differentiation factor 15 as predictor of mortality in acute reperfused ST-elevation myocardial infarction: insights from cardiovascular magnetic resonance. Heart. 2011;97(8):632-40.

17. Zhang S, Dai D, Wang X, et al. Growth differentiation factor-15 predicts the prognoses of patients with acute coronary syndrome: a meta-analysis. BMC Cardiovasc Disord. 2016;16:82.

18. Eggers KM, Lindahl B. Prognostic biomarkers in acute coronary syndromes: risk stratification beyond cardiac troponins. Curr Cardiol Rep. 2017;19(4):29.

19. van der Hoeven BL, Liem SS, Jukema JW, et al. Sirolimus-eluting stents versus bare-metal stents in patients with ST-segment elevation myocardial infarction: 9-month angiographic and intravascular ultrasound results and 12-month clinical outcome results from the MISSION! intervention study. J Am Coll Cardiol. 2008;51(6):618-26.

20. Liem SS, van der Hoeven BL, Oemrawsingh PV, et al. MISSION!: optimization of acute and chronic care for patients with acute myocardial infarction. Am Heart J. 2007;153(1):14.e1.

21. Van de Werf F, Ardissino D, Betriu A, et al. Management of acute myocardial infarction in patients presenting with ST-segment elevation. The Task Force on the Management of Acute Myocardial Infarction of the European Society of Cardiology. Eur Heart J. 2003;24(1):28-66.

22. Antman EM, Anbe DT, Armstrong PW, et al. ACC/ AHA guidelines for the management of patients with ST-elevation myocardial infarction: a report of the American College of Cardiology/American Heart Association Task Force on Practice Guidelines (Committee to Revise the 1999 Guidelines for the Management of Patients with Acute Myocardial Infarction). Circulation. 2004;110(9):e82-292.

23. Harrell FE Jr, Lee KL, Mark DB. Multivariable prognostic models: issues in developing models, evaluating assumptions and adequacy, and measuring and reducing errors. Stat Med. 1996;15(4):361-87.

24. Eggers KM, Kempf T, Lagerqvist B, et al. Growthdifferentiation factor-15 for long-term risk prediction in patients stabilized after an episode of nonST-segment-elevation acute coronary syndrome. Circ Cardiovasc Genet. 2010;3(1):88-96.

25. Lindholm D, James SK, Bertilsson $M$, et al. Biomarkers and coronary lesions predict outcomes after revascularization in non-ST-elevation acute coronary syndrome. Clin Chem. 2017;63(2):573-84.

26. Hagstrom E, James SK, Bertilsson M, et al. Growth differentiation factor-15 level predicts major 
bleeding and cardiovascular events in patients with acute coronary syndromes: results from the PLATO study. Eur Heart J. 2016;37(16):1325-33.

27. Lind L, Wallentin L, Kempf T, et al. Growth-differentiation factor-15 is an independent marker of cardiovascular dysfunction and disease in the elderly: results from the Prospective Investigation of the Vasculature in Uppsala Seniors (PIVUS) study. Eur Heart J. 2009;30(19):2346-53.

28. Wollert KC, Kempf T, Peter T, et al. Prognostic value of growth-differentiation factor-15 in patients with non-ST-elevation acute coronary syndrome. Circulation. 2007;115(8):962-71.

29. Xu J, Kimball TR, Lorenz JN, et al. GDF15/MIC-1 functions as a protective and antihypertrophic factor released from the myocardium in association with SMAD protein activation. Circ Res. 2006;98(3):342-50.

30. Salvagno GL, Pavan C. Prognostic biomarkers in acute coronary syndrome. Ann Transl Med. 2016;4(13):258.

31. Eggers KM, Kempf $\mathrm{T}$, Lind $\mathrm{L}$, et al. Relations of growth-differentiation factor-15 to biomarkers reflecting vascular pathologies in a populationbased sample of elderly subjects. Scand J Clin Lab Invest. 2012;72(1):45-51.
32. Kempf T, Horn-Wichmann R, Brabant G, et al. Circulating concentrations of growth-differentiation factor 15 in apparently healthy elderly individuals and patients with chronic heart failure as assessed by a new immunoradiometric sandwich assay. Clin Chem. 2007;53(2):284-91.

33. National Institute for Health and Care Excellence. Clinical guideline (CG187). Acute heart failure: diagnosis and management. London: NICE; 2014.

34. Monaghan PJ, Lord SJ, St John A, et al. Biomarker development targeting unmet clinical needs. Clin Chim Acta. 2016;460:211-9.

35. Bonaca MP, Morrow DA, Braunwald E, et al. Growth differentiation factor-15 and risk of recurrent events in patients stabilized after acute coronary syndrome: observations from PROVE IT-TIMI 22. Arterioscler Thromb Vasc Biol. 2011;31(1):203-10.

36. Ridker PM, Everett BM, Thuren T, et al. Antiinflammatory therapy with canakinumab for atherosclerotic disease. N Engl J Med. 2017;377(12):1119-31.

37. Daniels LB, Clopton P, Laughlin GA, Maisel AS, Barrett-Connor E. Growth-differentiation factor-15 is a robust, independent predictor of 11-year mortality risk in community-dwelling older adults: the Rancho Bernardo study. Circulation. 2011;123(19):2101-10. 